\title{
POLÍTICAS DE CONTROLE DO CÂNCER DE MAMA NO BRASIL
}

\author{
Thaislayne Nunes de Oliveira ${ }^{1}$ \\ Mônica de Castro Maia Senna²
}

Recebido em: 24/04/2021

Aprovado em: 26/05/2021

\begin{abstract}
Resumo: Este artigo tem como objetivo central examinar a trajetória histórica das políticas de controle do câncer de mama feminino no Brasil em diferentes momentos históricos. As primeiras intervenções públicas nessa direção no país surgiram em meados do século XX e visavam ao desenvolvimento do cuidado oncológico de maneira individual. A intensificação das medidas estratégicas para o seu controle ocorreu somente a partir dos anos 2000, com perceptível desenvolvimento de ações coletivas e incentivo à prevenção, de maneira a incidir diretamente no controle da doença. $\mathrm{O}$ estudo contou com pesquisa bibliográfica sobre a temática, associada à análise dos documentos oficiais, como: normativas, portarias e legislações nacionais. Os resultados demonstram avanços na estruturação do cuidado da doença, sobretudo pela implantação de políticas, programas e sistemas específicos. Tais avanços estratégicos contribuem positivamente para o controle de riscos e agravos da doença. Mas, apesar desses avanços, a realidade observada ainda permanece em certo descompasso, perceptível pelo elevado e crescente índice de mortalidade.
\end{abstract}

Palavras-chaves: políticas públicas de saúde; Sistema Único de Saúde; oncologia; câncer de mama; saúde da mulher.

\section{POLÍTICAS DE CONTROL DEL CÁNCER DE MAMA EN BRASIL}

Resumen: Este artículo pretende examinar la trayectoria histórica de las políticas de control del cáncer de mama femenino en Brasil en diferentes momentos históricos. Las primeras intervenciones públicas en este sentido en el país surgieron a mediados del siglo $\mathrm{XX}$ y pretendían desarrollar la atención oncológica de forma individual. La intensificación de las medidas estratégicas para su control sólo se produjo a partir de la década de 2000, con un notable desarrollo de acciones colectivas e incentivos para la prevención, con el fin de incidir directamente en el control de la enfermedad. El estudio contiene una investigación bibliográfica sobre el tema, asociada al análisis de los documentos oficiales, como: normativas, portarias y

\footnotetext{
${ }^{1}$ Assistente Social pela Universidade Federal Fluminense (UFF), Especialista em Saúde Pública (Hospital Universitário Antônio Pedro/UFF), Mestre e Doutoranda em Política Social pelo Programa de Estudos Pós Graduados em Política Social (UFF). Assistente Social do Núcleo de Apoio à Saúde da Família no Município do Rio de Janeiro. E-mail: thaiislayne@ hotmail.com e Orcid: https://orcid.org/0000-0002-7676-6825.

${ }^{2}$ Assistente Social. Sanitarista. Mestre e Doutora em Ciências - Saúde Pública. Professora Associada do Programa de Estudos Pós Graduados em Política Social e da Escola de Serviço Social da Universidade Federal Fluminense. Pesquisadora CNPq. E-mail: monica_senna@id.uff.br. Orcid: https://orcid.org/0000-0003-2161-7133.
} 
legislaciones nacionales. Los resultados demuestran los avances en la estructuración del cuidado de la enfermedad, sobre todo por la implantación de políticas, programas y sistemas específicos. Estos avances estratégicos contribuyen positivamente al control de los riesgos y agravamientos de la enfermedad. Pero, a pesar de estos avances, la realidad observada sigue estando en cierta desarmonía, perceptible por la elevada y creciente tasa de mortalidad.

Palabras clave: políticas públicas de salud; Sistema Único de Salud; oncología; neoplasias de la mama; salud de la mujer.

\section{BREAST CANCER CONTROL POLICIES IN BRAZIL}

Abstract: The central objective of this article is to examine the historical trajectory of policies to control female breast cancer in Brazil in different historical moments. The first public interventions in this direction in the country emerged in the mid-twentieth century and aimed at the development of oncological care in an individual way. The intensification of strategic measures for its control occurred only from the 2000s, with noticeable development of collective actions and incentives for prevention, in order to have a direct impact on the control of the disease. The study relied on bibliographic research on the theme, associated with the analysis of official documents, such as: norms, ordinances, and national legislation. The results show advances in the structuring of care for the disease, especially through the implementation of policies, programs, and specific systems. Such strategic advances contribute positively to the control of the risks and aggravations of the disease. But, despite these advances, the observed reality still remains in a certain disharmony, perceptible by the high and growing mortality rate. Keywords: public health policy; Unified Health System; oncology; breast neoplasms; women's health

\section{Introdução:}

O câncer é considerado pela Organização Mundial de Saúde (OMS) como uma questão de saúde pública, constituindo-se em uma das principais causas de mortalidade em escala global. Entre as mulheres, o câncer de mama é o mais incidente tanto no Brasil quanto no mundo. Conforme estimativas disponibilizadas pelo INCA (2019) espera-se a ocorrência de 66.280 casos novos de câncer de mama para cada ano do triênio 2020-2022, com um risco estimado de 61,61 casos a cada 100 mil mulheres.

Trata-se de uma doença com gênese multifatorial, podendo ser associada ao envelhecimento populacional, às mudanças ambientais e de hábitos de vida, como: obesidade, sedentarismo e ao consumo excessivo de álcool. Somado a isso, a localização tumoral interfere diretamente nos tipos de métodos preventivos e de tratamento. No caso do câncer de mama, a literatura indica como não sendo completamente evitável ou prevenível, mas, quando diagnosticado na fase inicial apresenta bom prognóstico e chances reais de recuperação. (OLIVEIRA, 2017). 
Apesar disso, dados disponibilizados pelo Atlas da mortalidade indicam que o câncer de mama é o que mais mata mulheres no Brasil, com taxa de 16,04 óbitos a cada 100 mil mulheres. Não se pode justificar o elevado número de óbitos ao também exorbitante número de casos novos, já que esse tipo de câncer está entre os menos letais. Então, considerando que a detecção precoce favorece as chances reais de recuperação, o elevado número de óbitos pode ser justificado pelas dificuldades de acesso oportuno ao diagnóstico e tratamento.

A complexidade do tratamento do câncer de mama, a exemplo de outras neoplasias, requer avanços científicos e tecnológicos, uma vez que ainda não se tem assegurada sua cura em definitivo. Junto a isso, como todas as outras doenças, o desenvolvimento das estratégias para o seu cuidado é inerente ao contexto de desenvolvimento da política de saúde brasileira, ou seja, a criação das medidas para seu controle perpassa por aspectos próprios do desenrolar da política pública de saúde.

O presente artigo examina as intervenções públicas adotadas para o controle do câncer de mama no Brasil em sua trajetória histórica. Busca-se compreender as tendências verificadas em momentos históricos específicos, associando-as ao contexto do país, em especial no que se refere à condução da política de saúde em cada momento.

Para tanto, o estudo serviu-se de pesquisa bibliográfica e levantamento documental. O levantamento documental consistiu na busca de legislações e normativas implantadas e relacionadas ao câncer de mama, além de busca de dados secundários relativos às estatísticas de saúde e estimativas relacionadas ao câncer de mama feminino, que foram localizadas nas plataformas DATASUS e INCA. Esta investigação subsidiou o momento seguinte, que consistiu na análise do material coletado relacionando a trajetória histórica das medidas evidenciando as alterações significativas na busca do controle do câncer de mama no Brasil.

\section{Eixos introdutórios sobre o câncer de mama no Brasil}

Mais do que um fenomeno biológico, ser mulher é uma construção social e, portanto, articula-se às particularidades próprias de cada sociedade e diferentes tempos históricos ${ }^{3}$. Do ponto de vista das políticas de saúde dirigidas às mulheres no Brasil as provisões do Estado direcionadas à saúde da mulher limitaram-se historicamente tão somente à maternidade (ou ao

\footnotetext{
${ }^{3}$ É importante salientar que as mudanças na vida das mulheres afetam a sua saúde. No caso do câncer de mama, a amamentação pode diminuir a chance de desenvolvimento da neoplasia mamária (INCA, 2009). Considerando que, paulatinamente, ocorreram mudanças importantes, tais como: a inserção da mulher o mercado de trabalho, o primeiro filho com idade mais avançada, número menor de filhos, ou até mesmo não ter filhos, são indicativos fundamentais, que inclusive precisam ser considerados no desenvolvimento das estratégias de prevenção.
} 
ciclo gravídico-puerperal). Essa tendência sofreu alterações somente no século XX, sobretudo por pressão do movimento das próprias mulheres, que buscavam atendimento às demandas inerentes às realidades vivenciadas para além do cuidado materno-infantil (BRASIL, 1983).

O Programa de Assistência Integral à Saúde da Mulher (PAISM), criado em 1983, foi precedido da participação ativa das mulheres em Confererências Mundiais, que clamavam pela necessidade de mudanças urgentes. Foi o start da abordagem da atenção integral em saúde, dando visibilidade a outras morbidades que poderiam acometer as mulheres, e nesse contexto evidenciou-se a necessidade de criar estratégias de cuidado para o câncer de mama. ${ }^{4}$

Apesar da década de 1980 ter iniciado o ensaio para a atenção ao câncer de mama, somente nos anos 2000 é que ocorreram incorporações que buscam efetivamente o seu controle. Junto a isso, o INCA aponta que o número de casos de câncer aumentou exponencialmente, em especial os cânceres de colo de útero e de mama.

Para o controle do câncer foram necessárias investigações científicas, que foram desenvolvidas nas formas de rastreio e estabelecimento de protocolos. Durante muitos anos, os exames ultrassom e mamografia foram considerados por muitos cientistas como forma de exposição para as mulheres e por isso poderiam ocasionar outras doenças, a recorrência aos exames foi disseminada a partir da década de 1990 (INCA, 2018; PORTO, TEIXEIRA e SILVA, 2013). Nesse sentido, o próprio progresso tecnológico associado às pesquisas científicas definiram as formas rastrear e tratar o câncer, e gradualmente desencadearam as orientações das ações para o incentivo a detecção precoce e controle da doença.

Gradativamente ocorreu o desenvolvimento das medidas organizativas do câncer, as quais foram concentradas cronologicamente no quadro 1, que sistematiza as principais evoluções normativas das políticas de controle do câncer de mama, sem pretender finalizar a discussão, mas subsidiar o momento seguinte, que consiste na análise temporal das principais alterações conquistadas 5 .

\section{Quadro 1: O controle do câncer de mama no Brasil}

\begin{tabular}{|c|l|}
\hline ANO & \multicolumn{1}{|c|}{ NORMATIVA/DESCRIÇÃO } \\
\hline 1957 & $\begin{array}{l}\text { Fundação das Pioneiras Sociais implantou o Hospital de Cancerologia da cidade do Rio } \\
\text { de Janeiro. }\end{array}$ \\
\hline
\end{tabular}

\footnotetext{
${ }^{4} \mathrm{~A}$ neoplasia de mama foi uma das doenças privilegiadas no PAISM por se tratar de uma doença com crescente número de casos e consequente aumento de óbitos. Junto disso, é importante considerar que a década de 1980 também foi fundamental a definição da saúde como política pública.

${ }^{5}$ Além das referidas legislações citadas no quadro 1 existem outras garantias legais que abarcam o gênero feminino, mas aqui utilizamos aquelas relacionadas ao campo da saúde, oncologia em geral e câncer de mama, que são aspectos centrais desta análise.
} 


\begin{tabular}{|c|c|}
\hline 1973 & $\begin{array}{l}\text { riação do Programa Nacional de Controle do Câncer, iniciativa pioneira com foco nos } \\
\text { inceres femininos por meio de ações de prevenção e oferta de mamografias e exames } \\
\text { Papanicolau. }\end{array}$ \\
\hline 1983 & cograma de Assistência Integral à Saúde da Mulher (PAISM). \\
\hline 1987 & $\begin{array}{l}\text { ançamento do Pró-Onco, programa que unia esforços do Ministério da Saúde e do } \\
\text { JAMPS para ampliar a informação e a prevenção dos cânceres femininos. O câncer de } \\
\text { ama foi contemplado por meio do incentivo ao autoexame das mamas e ao exame } \\
\text { ínico das mamas. }\end{array}$ \\
\hline 1988 & $\begin{array}{l}\text { rt. 196. A saúde é direito de todos e dever do Estado (Constituição Federal de } 1988 \text { - } \\
\text { F/1988). }\end{array}$ \\
\hline 1990 & $\begin{array}{l}\text { Lei } N^{\circ} 8.080 \text { Dispõe sobre as condições para a promoção, proteção e recuperação da } \\
\text { saúde. } \\
\text { Lei } N^{\circ} 8.142 \text { Dispõe sobre a participação da comunidade na gestão do SUS. }\end{array}$ \\
\hline 1993 & $\begin{array}{l}\text { Portaria } \mathrm{N}^{\circ} 170 / 1993 \text { estabeleceu as normas para credenciar os hospitais em } \\
\text { procedimentos para tratamento do câncer. }\end{array}$ \\
\hline 1997 & $\begin{array}{l}\text { Programa Viva Mulher: nacionalmente buscou o controle dos cânceres do colo do útero } \\
\text { e mama. }\end{array}$ \\
\hline 1998 & $\begin{array}{l}\text { Portaria } \mathrm{N}^{\mathrm{o}} 3.535 \text { dividiu e estabeleceu uma rede de tratamento de maneira } \\
\text { hierarquizada, com cadastramento de centros de atendimento em oncologia. }\end{array}$ \\
\hline 2004 & $\begin{array}{l}\text { lítica Nacional de Atenção Integral à Saúde da Mulher. } \\
\text { Conferência Nacional de Políticas para as Mulheres. } \\
\text { lano Nacional de Políticas para as Mulheres (PNPM). }\end{array}$ \\
\hline 2005 & $\begin{array}{l}\text { cológica (PNAO). } \\
\text { de Útero e Mama (2005-2007). }\end{array}$ \\
\hline 2006 & $\begin{array}{l}\text { orecoce do câncer de mama } \\
\text { Vida. }\end{array}$ \\
\hline 2007 & \\
\hline 2008 & $\begin{array}{l}\text { 11.664 Dispõe sobre a efetivação de ações de saúde que assegurem a prevenção, } \\
\text { ção, o tratamento e o seguimento dos cânceres do colo uterino e de mama. } \\
\text { a SAS/MS N } 779 / 08 \text { Implantação do Sistema de Informação sobre o Câncer de } \\
\text { (SISMAMA). }\end{array}$ \\
\hline 2009 & \\
\hline 2010 & nento: Recomendações para a redução da mortalidade do câncer de mama no \\
\hline 2011 & $\begin{array}{l}3^{\text {a }} \text { Conferência Nacional de Políticas para as Mulheres. } \\
\text { Lançamento do Plano de Fortalecimento da Rede de Prevenção, Diagnóstico e } \\
\text { Tratamento do Câncer, que enfatizou o controle do câncer de mama. E lançamento do } \\
\text { Plano de Ações Estratégicas para o enfrentamento das Doenças Crônicas Não } \\
\text { Transmissíveis no Brasil (2011 - 2022), que incluiu as ações de controle do câncer. }\end{array}$ \\
\hline 2012 & $\begin{array}{l}\text { Programa Nacional de Qualidade da Mamografia. } \\
\text { Lançamento da campanha nacional para reforço do diagnóstico precoce e das } \\
\text { recomendações para o rastreamento mamográfico. } \\
\text { Portaria GM/MS N } 2.30404 / 10 / 2012 \text { institui o Programa de Mamografia Móvel no } \\
\text { âmbito do SUS. } \\
\text { Lei } N^{\circ} 12.732 \text { Dispõe sobre o primeiro tratamento de paciente com neoplasia maligna } \\
\text { comprovada e estabelece prazo para seu início. }\end{array}$ \\
\hline 2013 & $\begin{array}{l}\text { Lei } \mathrm{N}^{\circ} 12.802 \text {. Dispõe sobre a obrigatoriedade da cirurgia plástica reparadora da mama. } \\
\text { Portaria No } 874 \text { que institui a Política Nacional para a Prevenção e Controle do Câncer } \\
\text { (em substituição a PNAO). }\end{array}$ \\
\hline 2014 & $\begin{array}{l}\text { Portaria } \mathrm{N}^{\circ} 189 \text { que estabelece incentivo financeiro no custeio dos serviços de } \\
\text { Referência para Diagnóstico de Câncer de Mama. }\end{array}$ \\
\hline 2015 & $\begin{array}{l}\text { Publicação das Diretrizes para a Detecção Precoce do Câncer de Mama no Brasil, } \\
\text { elaboradas com base na sistematização de evidências científicas. }\end{array}$ \\
\hline
\end{tabular}




\begin{tabular}{|c|l|}
\hline $\mathbf{2 0 1 7}$ & $4^{\circ}$ Conferência Nacional de Políticas para as Mulheres. \\
\hline $\mathbf{2 0 1 8}$ & $\begin{array}{l}\text { Lei } \mathrm{N}^{\circ} 13.522 \text { estabelece que serão desenvolvidas estratégias intersetoriais específicas } \\
\text { para mulheres com dificuldade de acesso às ações de saúde e controle dos cânceres do } \\
\text { colo uterino e de mama. }\end{array}$ \\
\hline $\begin{array}{l}\text { Portaria MS/SAS N } \\
\text { carcinoma de mama. }\end{array}$ \\
\hline $\mathbf{2 0 1 9}$ & $\begin{array}{l}\text { Documento: A situação do câncer de mama no Brasil: síntese dos dados dos sistemas } \\
\text { de informação. } \\
\text { Lei } \mathrm{N}^{\circ} \text { 13.896 Altera a Lei } \mathrm{n}^{\circ} \text { 12.732, de } 22 \text { de novembro de 2012, para que os exames } \\
\text { relacionados ao diagnóstico de neoplasia maligna sejam realizados no prazo de } 30 \\
\text { (trinta) dias, no caso em que especifica. }\end{array}$ \\
\hline
\end{tabular}

Fonte: Elaboração própria, 2021.

Com a análise do quadro 1 nota-se que a partir dos anos 2000 ocorreu uma enxurrada de garantias legais, fator primordial para redefinição do enfoque governamental, e conferindo, de forma inédita a organização das políticas do câncer no Brasil e o direcionamento para o enfoque nas medidas preventivas.

É inevitável o reconhecimento dos muitos avanços em relação à concepção e à abordagem do cuidado em saúde da mulher, bem como na construção de normativas e ferramentas. Mas, não podemos desconsiderar que as respectivas incorporações legais se desenvolvem dentro de um campo de disputa de interesses antagônicos, que são próprios dos conflitos das políticas públicas brasileiras.

\section{Elementos históricos e a situação brasileira do câncer de mama:}

Em um breve resgate histórico ${ }^{6}$ é possível constatar que as primeiras medidas sanitárias relacionadas ao câncer surgiram no início do século XX, atreladas exclusivamente ao tratamento, sobretudo por estarem vinculadas ao modelo de saúde curativo e campanhas sanitárias para combate de endemias, aspectos próprios do desenvolvimento da política de saúde brasileira. (BRAVO, 2001).

O conhecimento restrito acerca da etiologia, pela própria complexidade, influenciou na pouca ênfase da prevenção, enfocando inicialmente no tratamento individual. Nesse período, os índices de mortalidade eram baixos, mas devido à complexidade da doença já se tinha uma tendência da elevação dos números de mortes, como também por outros fatores transversais, como o envelhecimento, por exemplo. (OLIVEIRA, 2017).

\footnotetext{
${ }^{6}$ Não se pretende analisar todas as modificações históricas no que tange o câncer de mama, mas apontar as medidas relevantes.
} 
Barreto (2005) identifica que as primeiras medidas para cuidado do câncer iniciaram na década de 1920, sobretudo por meio das práticas filantrópicas. E o desenvolvimento do cuidado se intensificou no final da década de 1930, período em que o médico Mario Kroeff deu visibilidade a métodos cirúrgicos associados ao cuidado da doença. A complexidade da doença e do seu tratamento fez com que alguns médicos sanitaristas e cancerologistas recorressem ao exterior na busca de conhecimento científico para desenvolver os tipos de tratamento, aspecto evidenciado por Barreto (2005) e também categorizado em INCA (2018).

Em 1937 foi criado o primeiro Centro de Cancerologia, mas somente durante o governo de Juscelino Kubitschek iniciou incremento na saúde da mulher. É importante destacar que as décadas de 1930 e 1940 também foram marcadas pela participação da sociedade civil que impulsionaram a entrada do câncer na agenda política. Para tanto, evidencia-se o surgimento das Ligas Contra o Câncer.

Nesse sentido, com relação aos cânceres femininos, em 1957 a Fundação das Pioneiras Sociais implantou o Hospital de Cancerologia da cidade do Rio de Janeiro, que abarcava atendimento ambulatorial e simultânea realização de pesquisa clínica, principalmente buscando o desenvolvimento de medidas de prevenção ${ }^{7}$. As Pioneiras Sociais, então lideradas pela Primeira-dama do país, Sarah Kubitschek, e compostas por outras mulheres que se reuniram para traçar estratégias de ajuda coletiva. As pioneiras trabalhavam com obras sociais, principalmente dirigidas aos mais pobres, incluindo aspectos relacionados à saúde.

Segundo Temperini (2012), as Pioneiras Sociais gradativamente se configuraram como uma Fundação apoiada pelo próprio Estado. E por meio da Fundação das Pioneiras Sociais criaram-se diversos programas, entre eles "Saúde Sobre as Rodas", que teve como objetivo oportunizar o acesso à saúde para a população através de hospitais volantes. A autora também identifica que entre as prioridades das Pioneiras estavam o controle do câncer.

Esse breve resgate histórico nos indica que a "entrada" do câncer na agenda governamental ocorreu antes mesmo da estruturação da saúde como política pública. Percebese o padrão do Governo Federal junto à saúde, sobretudo ao assumir as responsabilidades junto à saúde pública, uma vez que as transferia para as Pioneiras ou oferecia através da compra dos serviços médicos.

\footnotetext{
${ }^{7}$ Em tempo, a implantação desta unidade foi sucedida do falecimento mãe da primeira dama - Sara Kubitschek, a Sra. Luiza Gomes de Lemos, que foi acometida de um câncer ginecológico. Posterior ao seu falecimento inaugurou-se a unidade Hospitalar de Cancerologia supracitada, que em época recebeu seu nome. Ressalta-se que esta unidade hospitalar originou o atual Hospital do Câncer III, situado no bairro de Vila Isabel, no Rio de Janeiro, trata-se de uma unidade exclusiva de tratamento de neoplasia mamária. (COSTA, 2014).
} 
Nas décadas seguintes 1960, 1970 e 1980 ocorreram mudanças importantes na luta pela incorporação da saúde como política pública e, por conseguinte, incidiram na configuração da abordagem e tratamento do câncer. Nesse período, no caso da população urbana assalariada o acesso ao tratamento precedia a vinculação com institutos de previdência, a chamada medicina previdenciária. Mas, na década de 1960 a Organização das Nações Unidas buscou promover e apoiar o desenvolvimento de políticas independente da vinculação trabalhista ou do local de moradia. Ainda assim, percebem-se vulnerabilidades no acesso ao diagnóstico e tratamento dos brasileiros.

No mesmo período, os países da América Latina firmaram compromisso com planejamento de ações integradas para o desenvolvimento social e econômico, reconhecendo o acesso à saúde como fundamental ao progresso do país (OLIVEIRA, 2017). Paralelamente as décadas de 1960/1970 foram marcadas pelo período ditatorial, que na área da saúde possibilitava ênfase na medicina curativa, com "articulação do Estado com os interesses do capital internacional, via indústrias farmacêuticas e de equipamento hospitalar" (BRAVO, 2001, s/p.). Nesse contexto, prevalecia o olhar individual da doença, reforçando as ações e tratamentos individualizados.

A subsequente transição democrática, e respectivos contextos: histórico, político e social foram determinantes no desenrolar da saúde e alcance da sua consolidação como política. Na década de 1980 a saúde alcançou dimensão de política pública vinculando-se à proposta da Reforma Sanitária, com a garantia do acesso igualitário e universal, e responsabilidade da execução pelo Estado. Essa garantia não findou os interesses difusos próprios da política pública, e inclusive desencadeou outros tipos de conflitos e disputas. ${ }^{8}$

É sabido que a consolidação da saúde, como política, ocorreu por meio da promulgação da Constituição Federal de 1988, que no seu art. 196 prevê a saúde como uma política pública de direito de todos e dever do Estado. Em seguida, ocorreu à implantação da Lei No 8.080/1990, que trata sobre as condições de promoção, proteção e recuperação de saúde, organizando o SUS e delimitando a organização da política. No mesmo ano, ocorreu a implantação da Lei $\mathrm{N}^{\circ}$ 8.142/1990 que complementa a legislação anteriormente citada e trata da participação da comunidade na gestão do SUS. Ambas as legislações são frutos da articulação do movimento sanitário que buscou formular uma política de saúde pública ampla e de acesso igualitário.

Destaca-se a importância dos movimentos sociais, que foram fundamentais na incorporação dos direitos sociais, principalmente durante a década de 1980, entre esses o

${ }^{8}$ Para aprofundamento ver Bravo (2001) e Bravo et al (2018). 
movimento feminista. As mulheres se organizavam na luta contra as desigualdades na relação de gênero e nos problemas de saúde que afetavam particularmente a população feminina (NUNES, 1991).

Na mesma época, a nível mundial o câncer despontava como uma doença com elevados números de casos novos e óbitos. Segundo o Instituto Brasileiro de Geografia e Estatística, a partir da década de 1990, o Brasil inicia uma transição da faixa etária populacional, a transição demográfica, que é inerente à transição epidemiológica, pois com o envelhecimento tem-se o aumento das doenças crônicas, e o câncer é uma delas. Junto disso, se tem um direcionamento das organizações mundiais para a estruturação de medidas para seu cuidado. (OLIVEIRA, 2017).

Em 1993, o marco legal que buscou normatizar o tratamento do câncer ocorreu por meio da publicação da Portaria $N^{\circ} 170 / 1993$, que estabeleceu as normas para credenciar os hospitais em procedimentos para tratamento do câncer. Tratou-se da primeira medida do sistema público, que buscou credenciar o sistema privado para tratar a doença.

Já em 1997 foi fundado o Programa Viva Mulher, que priorizou ações estratégicas para o combate ao câncer de mama. E, em 1998 uma nova portaria foi promulgada, a Portaria $\mathrm{N}^{\circ}$ 3.535/1998 que dividiu e estabeleceu uma rede de tratamento de maneira hierarquizada, com cadastramento de centros de atendimento em oncologia.

Essas portarias (Portaria No 170/1993 e Portaria $N^{\circ} 3.535 / 1998$ ) restringiram-se ao credenciamento de redes hospitalares para tratamento da doença, afirmando novamente o câncer enquanto uma doença pontual que requer intervenção medicamentosa e/ou abordagemtratamento individual. Paralelamente, segundo dados disponibilizados pelo INCA, gradativamente os números de câncer foram crescendo no Brasil, tendo em vista os fatores para desenvolvimento da doença, complexidade do tratamento e possibilidades de reincidir.

Cabe ressaltar que nos anos de 1990, ocorre o redirecionamento do papel do Estado, influenciado pelo período neoliberal. Tal ação tem influência direta na política de saúde, que nesta época perpassa por reformas, ou "contrarreformas" (BRAVO, 2001). Observa-se a transferência da responsabilidade do Estado para o mercado, principalmente por meio das Organizações Sociais, Organizações da Sociedade Civil de Interesse Público e também por Parcerias Público Privadas. Em tempo, essas propostas também estão presentes nos dias atuais e mantem-se, ou melhor, aprofundam-se os conflitos entre as propostas governamentais e definições asseguradas na carta constitucional e na Lei Orgânica da Saúde.

Evidencia-se o sucateamento da saúde pública e constante tentativa de privatização desse espaço, em meio a esse, "a recuperação de alguns dos pontos básicos da proposta 
governamental permite perceber que a execução da Reforma do Estado choca-se, radicalmente, com as conquistas sociais obtidas na Carta Constitucional de 1988" (IAMAMOTO, 2011, s/p.). Logo, o modelo inerente à Reforma Sanitária, afirmado na CF/1988, passa por tensões, devido ao projeto articulado ao mercado, comprometendo o avanço das estratégias previstas na CF/1988, SUS e nas legislações posteriores.

No que tange à discussão sobre o câncer, a partir dos anos 2000, há uma retomada na agenda política, corroborado por orientações da OMS. Para tanto, em maio de 2004 foi publicado a Política de Atenção Integral à Saúde da Mulher, que assegura o câncer de mama como prioridade na agenda governamental. No mesmo ano foi desenvolvido o primeiro Plano Nacional de Políticas para as Mulheres (PNPM), ${ }^{9}$ que também determinava o enfoque neste câncer, bem como as ações e estratégias para seu controle.

Nesse sentido, por meio dos parâmetros especificados pela própria OMS, o Brasil alterou o cuidado do câncer e instituiu uma política específica. Em 2005, foi publicada a Portaria N 2.439, a Política Nacional de Atenção Oncológica (PNAO), que afirmou o câncer como problema de saúde pública e estruturou a rede de atenção oncológica a ser implantada em toda unidade federada, estabelecendo parâmetros e diretrizes da atenção oncológica em consonância as diretrizes estabelecidas pelo SUS.

Ainda em 2005 foi elaborado um Plano de Ação para o Controle dos Cânceres de Colo de Útero e Mama (2005-2007) pelo Governo Federal, que assegurou diretrizes estratégias a fim de controlar os cânceres femininos mais incidentes em casos novos, por meio desta ocorreu à designação da prioridade da cobertura deste público, capacitações, incentivo as pesquisas e o rastreamento da doença por realização de mamografia.

As medidas aprovadas em meados dos anos 2000 foram essenciais, e incidiram positivamente na busca pelo controle do câncer de mama. Além disso, ainda ocorreram outras alterações importantes, como a publicação da Lei $N^{\circ} 11.664 / 2008$, que garante a realização de mamografias para todas as mulheres acima de 40 anos, bem como o encaminhamento a serviços

\footnotetext{
${ }^{9} \mathrm{O}$ primeiro PNPM foi produzido como resultado da $1^{\text {a }}$ Conferência Nacional de Políticas para as Mulheres. Fato que também ocorreu no II PNPM e no III PNPM, respectivamente em 2008 e 2011. Com isso, a discussão proporcionada nos espaços de Conferência resultava em produtos importantes, evidenciando a participação e o controle social. Cabe destacar que os planos foram sendo alargados a cada publicação, e todos direcionavam o câncer de mama como aspecto central. Nesse aspecto, a última medida do Governo Federal foi à realização do método avaliativo do plano, que foi elaborado em 2013 e buscava mapear as necessidades das mulheres no território nacional, o qual também determinou a avaliação sobre as questões relacionadas ao câncer de mama, mas que até o presente momento não apresentou os resultados alcançados.
} 
de maior complexidade para complementação diagnóstica e tratamento, quando houver necessidade ${ }^{10}$.

Em 2009, o INCA sediou o encontro que buscava definir as formas de rastreio e tratamento do câncer de mama, possibilitando como resultado a produção do documento "Parâmetros técnicos para o rastreamento do câncer de mama". Além do mais, na mesma época, foi criado o Sistema de Informação do Câncer de Mama (SISMAMA), que favorece a vigilância desta neoplasia.

Em 2012, o INCA publicou "Recomendações para a redução da mortalidade do câncer de mama no Brasill”, que apresentou as recomendações necessárias para o controle desse tipo de neoplasia. Os dados foram trabalhados conforme a realidade do Brasil, demonstrando que as orientações podem precisar de ajustes ao longo dos anos conforme fatos novos da realidade. Ao todo foram 14 recomendações do INCA.

No mesmo ano, buscando qualificar a realização dos exames de rastreio da doença, foi instituído o Programa Nacional de Qualidade em Mamografia, por meio da Portaria $\mathrm{N}^{\circ} 2.898$, que busca monitorar e avaliar os serviços de diagnóstico, submetido à Vigilância Sanitária e ao INCA, além de incidir em capacitações e qualificações para realização do exame. Essas ações corroboram para organização das ações de controle do câncer de mama, a fim de garantir a qualidade dos exames realizados.

Em 2013, as portarias $\mathrm{N}^{\circ} 2.439 / 2005$ e $\mathrm{N}^{\circ} 741 / 2005$ foram revogadas por meio das Portarias $N^{\circ} 874 / 2013$ e No 140/2014, que estabeleceram a Política Nacional para Prevenção e Controle do Câncer na Rede de Atenção à Saúde das pessoas com doenças crônicas no âmbito do SUS (PNPCC) e redefiniram os parâmetros dos estabelecimentos de saúde habilitados na atenção especializada em oncologia. Atualmente, a portaria $\mathrm{N}^{\circ} 874$ permanece em vigor e foi republicada no Anexo IX da Portaria de Consolidação No 2/GM/MS de 28 de setembro de 2017. E a Portaria No 140 foi substituída pela Portaria SAES/MS No 1.399, de 17 de dezembro de 2019, que redefiniu novamente os critérios de habitação dos estabelecimentos de saúde especializados em oncologia.

Mais do que uma narrativa dos acontecimentos históricos, este estudo busca ratificar a importância de cada normativa, sobretudo por reconhecer nas respectivas análises documentais as estratégias dos riscos e agravos para vigilância e controle do câncer. Por exemplo, a própria

\footnotetext{
${ }^{10}$ Cabe ressaltar, que a referida legislação foi alterada em 2017 , por meio da Lei $\mathrm{N}^{\circ} 13.522$, com respectivas adequações às necessidades das mulheres que enfrentem dificuldade de acesso às ações de saúde, por motivos sociais, geográficos ou culturais, recomendando então a realização de busca ativa e desenvolvimento de estratégias necessárias.
} 
criação da PNPCC prevê estratégias e reconhece a importância do cuidado na fase inicial da doença, salientando aspectos do cuidado integral, com a articulação da promoção de saúde e da prevenção. Nesse sentido, a política prevê ainda o enfoque nas medidas de prevenção para alcançar o controle da doença.

Para tanto, evidencia a importância da assistência ao paciente oncológico, como não exclusiva da alta complexidade, afirmando a centralidade da Atenção Primária à Saúde como geradora/ coordenadora do cuidado, ainda que o acesso à confirmação do diagnóstico seja pela via da urgência e emergência. Reconhecendo assim a complexidade da doença e a necessidade de articulação nas esferas para seu controle.

Destaca-se que a política de prevenção prevê ainda a modificação na abordagem da doença, tendo em vista o enfoque da prevenção e do seu controle, reafirmando a importância do acesso universal e igualitário às ações e serviços. Reconhecendo aqui a articulação entre a promoção de saúde e a prevenção, a fim de subsidiar o monitoramento, assim como estabelece o SUS.

Em termos financeiros, a política de prevenção busca reduzir o custo do tratamento. Então, a detecção precoce da doença apresenta-se como vantajosa. Cabe salientar que apesar de especificar as fontes de financiamento, a política não delimita estimativa e/ou percentual, o que pode ser uma fragilidade na garantia de sua implementação. Apesar disso, em 2014, ocorreu à publicação da Portaria No 189/2014 que estabelecia o incentivo financeiro no custeio dos serviços de Referência para Diagnóstico de Câncer de Mama, posiciona percentuais de incremento a cada procedimento realizado em sua detecção.

Além disso, ocorreu aumento no número da realização de mamografia, sobretudo de 2012-2017, contudo, apesar do aumento na realização de exames, ainda persistem iniquidades no acesso, apontadas em outros estudos nacionais e internacionais que, inclusive, sinalizam mulheres, na faixa etária indicada, que nunca realizaram mamografia (VILLAR et al, 2015). A este respeito, Villar et al (idem) realiza estudo no Rio de Janeiro, e apresenta a discussão acerca dos mamógrafos. SUS e não SUS distribuíram-se desigualmente nas regiões do estado, apresentando mulheres dentro da faixa etária recomendada para rastreamento da doença que nunca fizeram mamografia. Os pesquisadores indicam a necessidade de instalar novos mamógrafos, realizar manutenção dos existentes, priorizarem estratégias de regionalizar os serviços ou utilizar mamógrafos móveis e, ainda destacam que tais fragilidades implicam no diagnóstico tardio e prognóstico das mulheres.

Ainda assim, não se pode negar que as recomendações são positivas e buscam o controle do câncer de mama no Brasil, sobretudo compreendendo a realidade que a mulher está inserida 
na sociedade e todas as garantias legais necessárias para provimento do seu cuidado. Mas, cabe refletir: se os números de óbitos permanecem elevados e crescentes, as estratégias regulamentadas são suficientes para controlar os casos de câncer no Brasil? A análise do material demonstra que sim, todo esse contexto é notadamente como um avanço acertado, pois as medidas inéditas corroboram positivamente nas formas de cuidado da doença. Porém, percebe-se a fragilidade na sua execução plena, fator que incidi diretamente na continuidade dos crescentes números de óbitos.

Reiterando a complexidade do câncer de mama, o INCA publicou um documento orientador "A situação do câncer de mama no Brasil: síntese dos dados dos sistemas de informação", que foi lançado em 2019 e expõe uma síntese sobre a situação nacional. O documento ratifica o câncer de mama como um desafio no cenário atual, apresenta as linhas de cuidado e reconhece os limites das estratégias que ainda precisam ser ofertadas para o controle da doença no Brasil. Vale ressaltar que o documento também evidencia que as principais medidas de controle do câncer de mama ocorreram a partir da década de 2000.

Contudo, cabe destacar que desde o impeachment da Presidente Dilma, em dezembro de 2016, com exceção da Lei $N^{\circ}$ 13.522/2017 e do documento acima citado, não se realizou publicação de novos planos, legislações específicas ou outras normativas desta área. A ascensão de Michel Temer à Presidência da República após o Golpe jurídico-parlamentar que, levou ao impedimento de sua antecessora, culminou em ações que fragilizaram em muito a condução desta política. Ainda em sua posse, Temer foi criticado pela ausência da representatividade feminina em seu escalão de ministros. Em seguida, rebaixou o Ministério da Mulher ao status de Secretaria vinculada ao Ministério dos Direitos Humanos.

Cabe ressaltar a aprovação de medidas transversais que influíram diretamente no desenvolvimento da política de saúde da mulher, com destaque para a Emenda Constitucional $n^{\circ}$ 95/2016, que congelou os gastos públicos federais e investimento nas áreas da saúde e educação por 20 anos. Trata-se de um imenso retrocesso, impedindo, inclusive, a continuidade do que estava assegurado pela gestão anterior. O contexto foi e ainda é de dúvidas e incertezas com relação ao futuro da política pública de saúde, com reflexos importantes a condução da política de oncologia.

Esse quadro parece se aprofundar ainda mais no governo de Jair Bolsonaro, que assume a Presidência do país em janeiro de 2019, com uma base de apoio heterogênea, mas altamente caracterizada pelo conservadorismo moral e religioso e pela associação a grupos econômicos ultraliberais. Em seus primeiros anos de gestão, assiste-se a um processo de desmonte das 
políticas e direitos sociais duramente conquistados pela sociedade brasileira, em que a atenção à saúde da mulher não se encontra imune.

Na nova estrutura de governo, foi criado o Ministério da Mulher, da Família e dos Direitos Humanos, tendo à frente uma das poucas representantes femininas na composição ministerial. De vinculação religiosa, a ministra tem defendido, entretanto, uma pauta bastante conservadora em defesa da "tradicional família brasileira", repudiando até mesmo o uso do termo gênero e reforçando a clara definição de papeis sexuais historicamente consolidados.

Essa retomada da moralização conservadora e incentivo a campanhas antifeministas se dá em um contexto marcado pelo aumento de casos de violência contra a mulher e pela adoção de um conjunto de proposições governamentais que tendem a aumentar as desproteções sociais e representar um abandono à própria sorte. Com a ausência da discussão crítica e ampliada sobre a temática, há uma espécie de campo arenoso, onde não há certezas, pairando dúvidas sobre a manutenção e o futuro das políticas outrora alcançadas.

No que tange especificamente à política de controle do câncer, recentemente foram disseminadas informações, massivamente transmitida pela mídia televisa, sobre a restrição da fabricação e fornecimento de alguns medicamentos para o tratamento oncológico. Informação desmentida por representantes do Governo, que afirmam que a população não será afetada. Contudo, a disseminação desse tipo de notícia fragiliza, ainda mais, pacientes oncológicos e coaduna com a incerteza no desenvolvimento das políticas anteriormente conquistadas.

Nesta análise percebeu-se a modificação no acesso às informações sobre prevenção e foco no controle do câncer de maneira positiva, sobretudo nas ações de vigilância da doença. Por tudo isso, tem que se reconhecer o avanço no sentido das normativas e ferramentas que corroboram a conjuntura do câncer de mama.

Por tais razões, evidencia-se a necessidade de discutir a prevenção e promoção da saúde e a relevância dos determinantes sociais e seus impactos nos números de casos novos de câncer no Brasil e a nível mundial. Assim, constata-se o avanço na estruturação das legislações, além da implantação de programas e sistemas relacionados ao câncer de mama, que favorecem a detecção precoce.

Contudo, apesar da existência dos programas, dos sistemas e da política, a realidade observada ainda permanece em certo descompasso, perceptível pelo número de casos que evoluem a óbito e fragilizados pelo contexto atual da política, como exposto no decorrer deste trabalho. Com isso, observa-se que ainda não se tem estrutura capaz de subsidiar o número de atendimentos necessários no momento oportuno, corroborando alto índice de mortalidade. 


\section{Considerações finais}

A atenção à saúde da mulher se modificou nas últimas quatro décadas, mas, sobretudo na década de 2000, na qual ocorreram incorporações legais inéditas. Destaca-se a inclusão das requisições dos movimentos das mulheres, que buscavam a ampliação da saúde para além do cuidado materno-infantil, a fim de privilegiar o cuidado global da mulher em suas reais necessidades, discussão iniciada com a criação do PAISM, em 1983. Além disso, é importante salientar a construção do programa, política, planos e demais medidas que alinharam o cuidado em saúde da mulher, mesmo que de forma gradual, pois estas ações possibilitaram conquistas históricas imensuráveis.

No que tange o câncer de mama, faz-se necessário considerar que o adoecimento por esse diagnóstico repercute de diversas formas e em diferentes esferas da vida das mulheres. Aliado à debilidade física, à adoção de procedimentos invasivos e dolorosos e até ao risco de morte, encontra-se um conjunto de alterações no modo de andar a vida dessas mulheres, que passam desde a rotina diária até suas relações familiares, de trabalho e sociais. Por isso, faz-se necessário o analisar esse diagnóstico a partir de elementos que configuram o acesso ao tratamento oportuno e recuperação da doença.

$\mathrm{Na}$ atualidade, persiste como sendo o tipo de neoplasia mais causadora de morte no mundo entre as mulheres e permanecem elevadas as estimativas de números de casos novos, e consequentemente esse crescimento reflete na maior demanda por tratamento. Logo, compreende-se que uma doença de alta complexidade requer articulação junto à rede de serviços.

Evidenciam-se os progressos em torno da abordagem da neoplasia de mama na agenda política, com maior ênfase a partir dos anos 2000. Com desenvolvimento e estruturação das legislações e implementação de política, programas e sistemas específicos, que tendem a favorecer a rastreamento da doença e a detecção precoce, e consequentemente incidir positivamente no controle da doença. Mas, também foram salientadas as fragilidades impostas pelo atual cenário político brasileiro e sua implicação na conjuntura na política de saúde e na situação do câncer de mama.

Sendo assim, a realidade brasileira identificada ainda permanece em descompasso, sobretudo ao analisarmos os números de óbitos pela doença. Assim, concluímos apontando a necessidade da execução plena das garantias legais, da manutenção e continuidade das políticas sociais estatais, considerando as condições de vida das mulheres, na perspectiva do direito à 
saúde integral, assegurado na carta constitucional de 1988 e em leis posteriores abordadas no decorrer deste estudo.

\section{Referências bibliográficas}

BARRETO, Eliana Maria Teixeira. Acontecimentos que fizeram a história da oncologia no Brasil. Revista Brasileira de Cancerologia, Rio de Janeiro. 2005; 51(3): 267-75.

BRASIL. Constituição Fe(exceto câncer de pele não melanoma)deral de 1988.

BRASIL. Programa de Assistência Integral á Saúde da Mulher: bases de ação programáticaMinistério da Saúde. - Brasília, Centro de Documentação do Ministério da Saúde, 1983.

BRASIL. Lei No 8.080, de 19 de setembro de 1990.

BRASIL. Lei $\mathrm{N}^{\circ} 8.142$, de 28 de dezembro de 1990.

BRASIL. Portaria No 170, 11 de abril de 1993.

BRASIL. Portaria No 3.535, de 2 de setembro de 1998.

BRASIL. Política Nacional de Atenção Integral à Saúde da Mulher: princípios e diretrizes / Ministério da Saúde, Secretaria de Atenção à Saúde, Departamento de Ações Programáticas Estratégicas. - Brasília: Ministério da Saúde, 2004.

BRASIL. Plano Nacional de Políticas para as Mulheres. Brasília: Secretaria de Políticas para as Mulheres, Brasília, 2004.

BRASIL. Portaria $\mathrm{N}^{\circ} 2.439$, de 8 de dezembro de 2005.

BRASIL.Lei No 11.664, de 29 de abril de 2008.

BRASIL. Plano Nacional de Políticas para as Mulheres. Brasília: Secretaria de Políticas para as Mulheres, Brasília, 2004.

BRASIL. Lei N ${ }^{\circ} 12.802$, de 24 de abril de 2013.

BRASIL. Portaria $\mathrm{N}^{\circ} 874$, de 16 de maio de 2013.

BRASIL. Portaria $\mathrm{N}^{\circ} 2.898$, de 28 de novembro de 2013.

BRASIL. Portaria No 189, de 31 de janeiro de 2014.

BRASIL. Emenda Constitucional N ${ }^{\circ}$ 95, de 15 de dezembro de 2016.

BRASIL. Lei $\mathrm{N}^{\circ}$ 13.522, de 27 de novembro de 2017.

BRAVO, Maria Inês Souza; PELAEZ, Elaine Junger; PINHEIRO, Wladimir Nunes. "As contrarreformas na política de saúde do governo Temer". Argum, Vitória, v 10, n.1, p. 923, jan./abr. 2018.

BRAVO, Maria Inês Souza. "Política de Saúde no Brasil", Rio de Janeiro: 2001.: Disponível em: <www.fnepas.org.br/pdf/servico_social_saude/texto1-5.pdf> Acesso em 20 de março de 2021.

COSTA, Andreia Martins da. "A ORGANIZAÇÃO DA ATENÇÃO AO CÂNCER DE MAMA NOS MUNICÍPIOS: estudo de um sistema local de saúde.” Dissertação (Mestrado em Política Social) - Universidade Federal Fluminense, Escola de Serviço Social, 2014.

IAMAMOTO, Marilda Vilela. O Serviço Social na contemporaneidade: trabalho e formação. São Paulo: Cortez, 2011.

Instituto Nacional do Câncer (Brasil). Plano de Ação para o Controle dos Cânceres de Colo de Útero e Mama (2005-2007). Instituto Nacional do Câncer, Rio de Janeiro: INCA, 2005.

Instituto Nacional do Câncer (Brasil). Sumário Executivo. Políticas e Ações para Prevenção do Câncer no Brasil. Alimentos, Nutrição e Atividade Física. Rio de Janeiro: INCA, 2009.

Instituto Nacional do Câncer (Brasil). Recomendações para redução da mortalidade por câncer de mama no Brasil: balanço 2012. - Rio de Janeiro: Inca, 2012.

Instituto Nacional do Câncer (Brasil). Parâmetros técnicos para o rastreamento do câncer de mama. Rio de Janeiro, 2009. 
Instituto Nacional do Câncer (Brasil). O controle do câncer de mama no Brasil: Trajetórias e controvérsias - Rio de Janeiro: INCA; Fiocruz, 2018.

Instituto Nacional do Câncer (Brasil). Viva Mulher 20 anos: história e memória do controle do câncer do colo do útero e de mama no Brasil: catálogo de documentos - Rio de Janeiro: INCA, 2018.

Instituto Nacional do Câncer (Brasil). Estimativa 2020. Incidência do Câncer no Brasil. Rio de Janeiro: INCA, 2019.

Instituto Nacional do Câncer (Brasil). A situação do câncer de mama no Brasil: síntese dos dados dos sistemas de informação - Rio de Janeiro: INCA, 2019.

NUNES, Silvia Alexim. A medicina social e a questão feminina. Physis: Revista de Saúde Coletiva, Rio de Janeiro, 1991; 1: 49-76.

OLIVEIRA, Thaislayne Nunes de. "Proteção social dirigida às mulheres com câncer de mama: um estudo exploratório". Dissertação de Mestrado, Universidade Federal Fluminense, Rio de Janeiro, 2017.

PORTO, Marco Antônio Teixeira; TEIXEIRA, Luiz Antônio e SILVA, Ronaldo Corrêa Ferreira da. "Aspectos Históricos do Controle do Câncer de Mama no Brasil". Revista Brasileira de Cancerologia 2013; 59(3): 331-339 Disponível em: <rbc.inca.gov.br/site/arquivos/n_59/v03/pdf/03-artigo-aspectos-historicos-controle-cancermama-brasil.pdf $>$ acesso em 15 de março de 2021.

TEMPERINI, Rosana Soares de Lima. "Fundação das Pioneiras Sociais: Contribuição Inovadora para o Controle do Câncer do Colo do Útero no Brasil, 1956-1970” Revista Brasileira de Cancerologia 2012; 58(3): 339-349. Disponível em: <rbc.inca.gov.br/revista/index.php/revista/article/view/582/359> acessado em 10 de Janeiro de 2021.

VILLAR, Vanessa Cristina Felipe Lopes; SOUZA, Claudia Teresa Viana de; DELAMARQUE, Elisabete Vianna; SETA, Marismary Horsth de. Distribuição dos mamógrafos e dos exames mamográficos no estado do Rio de Janeiro, 2012 e 2013. Epidemiol. Serv. Saúde. 2015; 24(1): 115-22.

\section{Sites:}

portalms.saude.gov.br/ - Acesso em 10 de janeiro de 2021.

www.inca.gov.br/ - Acesso em 10 de janeiro de 2021.

www.inca.gov.br/rbcl - Acesso em 14 de mar. de 2021.

www.scielo.org/php/index.php - Acesso em 14 de mar. de 2021.

www.capes.gov.br/ - Acesso em 14 de mar. de 2021.

datasus.saude.gov.br/ - Acesso em 16 de mar. de 2021.

mortalidade.inca.gov.br/MortalidadeWeb/ - acesso em 10 de dez. de 2020. 